Emérita Sátiro Opaleye ${ }^{1}$

Helena lutéscia luna Coelho ${ }^{2}$

LaVÍNIA SCHÜLER-FACCIN $]^{3}$

Paulo César de Almeida ${ }^{4}$

Elaine Cavalcante dos Santos ${ }^{5}$

Ana Júla Velozo Ribeiro ${ }^{5}$

FabrícIo da SIIIA COSTA ${ }^{6}$

\section{Avaliação de riscos teratogênicos em gestações expostas ao misoprostol}

\author{
Evaluation of the teratogenic risks in gestations exposed to misoprostol
}

Artigo original

\section{Palavras-chave \\ Misoprostol \\ Teratogênios \\ Anormalidades congênitos/etiologia \\ Aborto induzido \\ Abortivos nãoesteroides \\ Fatores de risco \\ Keywords \\ Misoprostol \\ Teratogens \\ Congenital abnormalities/etiology \\ Abortion, induced \\ Abortifacient agents, nonsteroidal \\ Risk factors}

\section{Resumo}

OBJETIVOS: a tentativa de aborto mal sucedida com o uso do misoprostol (Cytotec $\left.{ }^{\circledR}\right)$ sem indicação médica tem sido associada a malformações congênitas. Este estudo teve por objetivo identificar, em recém-nascidos malformados e controles normais, a frequência de exposição ao misoprostol e o espectro de malformações associadas. MÉTODOS: estudo de caso-controle desenvolvido em 2005 nas quatro principais maternidades públicas de Fortaleza (CE). Através de busca ativa diária, foram identificados recém-nascidos com diagnóstico de malformação fetal (caso) e controles saudáveis de mesmo sexo nascidos em seguida na mesma maternidade (pareamento 1:1). A amostra foi de 252 parturientes entrevistadas por equipe treinada utilizando questionário estruturado com base no Estudo Colaborativo Latino-Americano de Malformações Congênitas (ECLAMC). Além de abordar questões sociodemográficas e histórico familiar de malformação, o questionário objetivou identificar exposições diversas durante a gestação, incluindo o misoprostol. A análise bivariada com teste do $\chi^{2}$ comparou os grupos quanto às características e fatores associados à malformação e foi calculada a Odds Ratio para verificar a razão de chances de o Grupo Caso apresentar malformação em relação ao Grupo Controle com relação à exposição ao misoprostol. RESULTADOS: não houve diferenças significativas entre os grupos caso e controle quanto à maioria dos fatores de riscos investigados para malformações. O relato de tentativa de aborto foi de $6,8 \%$, havendo uma maior exposição ao misoprostol durante a gestação em neonatos malformados comparados a saudáveis, Odds Ratio $(O R)=3,65$ (IC95\%=0,74-17,91). $\bigcirc$ espectro de malformações encontradas entre os recém-nascidos expostos ao misoprostol foi compatível com a literatura, como os decorrentes de defeitos do tubo neural e disrupção vascular. CONCLUSÕES: os achados deste estudo, apesar de não apresentarem significância estatística, sugerem que os fetos expostos ao misoprostol apresentam uma tendência a maior risco de defeitos congênitos comparados aos não-expostos. Outras investigações devem ser incentivadas para que se identifique melhor o dano causado pela utilização indevida do misoprostol, principalmente em países onde o controle de medicamentos é ineficaz.

\section{Abstract}

PURPOSE: failed attempted abortions with the use of misoprostol $\left(C_{\text {- }}\right.$ totec $\left.{ }^{\circledR}\right)$ without medical indication have been associated with the occurrence of congenital malformations. The objective of the present study was to identify, in newborns with malformations and in normal controls, the frequency of exposure to misoprostol and the spectrum of associated malformations. METHODS: this was a case-control study involving a daily survey at four public maternities in Fortaleza (CE) for the identification of newborns with malformations and paired controls $(1: 1)$ during the period from July to November 2005. The sample comprised 252 parturients interviewed by a trained team by means of a structured questionnaire based on the Latin American Collaborative Study of Congenital Malformations (Estudo Colaborativo Latino-Americano de Malformações Congênitas, ECLAMC). The questionnaire was used to obtain sociodemographic data and a family history of malformations, as well as to identify diverse forms of exposure during pregnancy, including misoprostol. Bivariate analysis and the chi-square test were used to compare cases and controls regarding their characteristics and factors associated with malformation, and the Odds Ratio was calculated to determine the chance of the Case Group to present malformations as compared to the Control Group after exposure to misoprostol. RESULTS: there were no significant differences between groups regarding most of the risk factors for malformations investigated.
Correspondência:

Departamento de Farmácia Centro de Ciências da Saúde Rua Capitão Francisco Pedro, 1.210 - Rodolfo Té́filo CEP 60431-327 - Fortaleza (CE), Brasil Fone-/Fax: (85) 3366-8058

Recebido

$30 / 11 / 09$

Aceito com modificacões

\section{Centro de Ciências da Saúde da Universidade Federal do Ceará - UFC - Fortaleza (CE), Brasil.}

' Mestrado em Ciências Farmacêuticas com Área de Concentração em Farmácia Clínica, Universidade Federal do Ceará - UFC - Fortaleza (CE), Brasil.

2 Professora Titular da Faculdade de Farmácia, Odontologia e Enfermagem, Universidade Federal do Ceará - UFC - Fortaleza (CE), Brasil. ${ }_{3}^{3}$ Professora Associada do Departamento de Genética da Universidade Federal do Rio Grande do Sul - UFRG - Porto Alegre (RS), Brasil.

4 Professor Adjunto do Mestrado Acadêmico em Saúde Pública, Universidade Estadual do Ceará - UFC - Fortaleza (CE), Brasil.

${ }_{5}^{5}$ Acadêmicos de Medicina da Universidade Estadual do Ceará - UFC - Fortaleza (CE), Brasil.

- Professor Adjunto do Mestrado Acadêmico em Saúde Pública e do Programa de Doutorado em Saúde Coletiva da Universidade Estadual do Ceará - UFC - Fortaleza (CE), Brasil

Não há conflito de interesse. 
Attempted abortion was reported by $6.8 \%$ of the mothers, with a higher exposure to misoprostol during pregnancy resulting in a greater proportion of malformed newborns, Odds Ratio $(\mathrm{OR})=3.65(95 \% \mathrm{Cl}=0.74-17.91)$. The spectrum of congenital defects encountered with exposure to misoprostol included defects of the central nervous, musculoskeletal, urogenital and cardiovascular systems, in agreement with literature data. CONCLUSION: the findings of this study suggest that fetuses exposed to misoprostol tend to be at higher risk of developing congenital malformations in comparison to non-exposed fetuses. Other studies should be encouraged for a better identification of the damage caused by the improper use of misoprostol, especially in countries where the control of medication is inadequate.

\section{Introdução}

No Brasil, o aborto é amparado por lei somente em casos de risco de morte para a mãe ou de estupro. Apesar de proibido, é prática conhecida em nosso país, com estimativas divergentes, que variavam na década de 1990 entre 300 mil e 3,3 milhões de abortos ilegais realizados a cada ano. Dados mais recentes indicam uma redução no número de internações por abortamento registradas pelo Sistema Único de Saúde entre 1992 e 2005 (de 344.956 para 250.447) abrangendo todos os grupos etários, de 15 a 49 anos $^{1}$. No entanto, a falta de estatísticas oficiais e de estudos com base populacional dificulta uma avaliação da magnitude do problema do aborto no Brasil. Os poucos dados disponíveis são obtidos por meio de pesquisas hospitalares, subestimando a população que recorre à prática.

Por outro lado, a redução nas internações hospitalares por abortamento nos últimos anos pode ser explicada pela utilização de métodos mais seguros, que não necessariamente obrigam a mulher a utilizar cuidados médicos. Um dos principais agentes referidos como a escolha para o aborto autoinduzido é o misoprostol $\left(\right.$ Cytotec $\left.^{\circledR}\right)$, utilizado com este fim desde seu lançamento comercial no Brasil, em meados da década de $1980^{2}$.

Atualmente, com seu uso restrito à rede hospitalar no Brasil, o misoprostol é adquirido de forma clandestina, sendo uma alternativa para gestações indesejadas pela acessibilidade, segurança de uso e menor custo, segundo avaliação das próprias usuárias ${ }^{3}$. A experiência como abortivo rendeu aplicações do misoprostol na obstetrícia. Utilizado isoladamente ou em associação à mifepristona e metotrexato, via oral ou vaginal, em vários ensaios clínicos foi avaliada a eficácia abortiva do misoprostol ${ }^{4,5}$. Usado de forma clandestina e sem assistência médica, o misoprostol tem sua efetividade como abortivo questionada, uma vez que nos ensaios clínicos a porcentagem de falhas ocorre em até $10 \%$ dos $\operatorname{casos}^{6}$. Dessa forma, a gestação pode não ser interrompida e o feto é exposto a um risco de eventos adversos que ainda não é amplamente conhecido ${ }^{7}$.

Vários relatos de casos publicados nos últimos anos sugerem uma associação entre a exposição intrauterina ao misoprostol e malformações congênitas (MF). A principal delas é a sequência de Möebius, uma condição clínica rara caracterizada por paralisia de nervos cranianos com comprometimento ocular ou facial uni ou bilateral, frequentemente associada a MF musculares e ósseas nos membros superiores ou inferiores ${ }^{8,9}$. Uma meta-análise recente avaliou estudos de caso-controle envolvendo 4.899 casos e 5.742 controles, e verificou o aumento do risco de anomalia congênita associada ao misoprostol e especificamente de sequência de Möebius e defeitos de redução transversa de membros ${ }^{7}$.

Estudos prospectivos, no entanto, apresentam resultados controversos quanto a este risco. Em estudo com coorte, não foi encontrado efeito teratogênico ao comparar 86 grávidas expostas ao misoprostol com 86 não-expostas ${ }^{10}$. Por outro lado, em uma coorte de 4.862 mulheres grávidas observou um risco 2,6 vezes maior no grupo exposto ao misoprostol de o recém-nascido $(\mathrm{RN})$ evoluir com $\mathrm{MF}$ congênitas quando comparado aos não-expostos ${ }^{11}$.

Nesse contexto, o presente estudo objetivou ampliar o conhecimento acerca da exposição fetal ao misoprostol por meio da comparação de recém-nascidos malformados e controles normais quanto à frequência de exposição ao misoprostol nas principais maternidades de Fortaleza.

\section{Métodos}

Foi realizado um estudo de caso-controle nas quatro principais maternidades públicas de Fortaleza, Ceará: Maternidade Escola Assis Chateubriand, Hospital Geral Dr. César Cals, Hospital Geral de Fortaleza e Hospital Distrital Gonzaga Mota de Messejana. Os hospitais foram selecionados por responderem juntos por mais de metade dos partos realizados por ano no município ${ }^{12}$ e três deles são hospitais de referência para resolução de partos com diagnóstico pré-natal de MF. A coleta de dados se deu no período de julho a novembro de 2005.

Os critérios de inclusão e exclusão seguiram o método do Estudo Colaborativo Latino-Americano de Malformações Congênitas (ECLAMC) ${ }^{13}$. Considerou-se caso o neonato vivo ou morto com peso igual ou superior a $500 \mathrm{~g}$, independentemente do sexo, produto ou não de gestação única, diagnosticado com MF congênita no pré-natal ou durante o período neonatal até a sua alta hospitalar, nascido nas instituições da pesquisa no decorrer do estudo. Foi considerado controle o primeiro recém-nascido vivo ou morto, sem $\mathrm{MF}$ e de mesmo sexo, nascido no mesmo hospital imediatamente após a detecção de um caso. $\mathrm{Na}$ impossibilidade de se coletar os dados do primeiro $\mathrm{RN}$ após o caso, considerava-se o primeiro $\mathrm{RN}$ nascido a partir das oito horas do dia seguinte. Esta definição fornece um Grupo Controle pareado um a um por sexo, lugar e período de nascimento com o grupo de MF. 
Antes do início da coleta, os hospitais foram visitados para apresentação do estudo e treinamento da equipe responsável pela notificação dos casos, havendo também a possibilidade de os hospitais notificarem casos de forma espontânea. A busca de RN que preenchiam os critérios de inclusão da amostra se dava através de telefonemas diários para cada um dos hospitais. Ao ser constatado um caso, ou mesmo uma suspeita, um entrevistador era acionado, seguindo-se escala de rodízio. A equipe era formada por dez estudantes de medicina previamente treinados por geneticista clínica e pela farmacêutica responsável pelo estudo. O entrevistador se deslocava até o hospital de origem para realizar entrevista com a mãe. Empregava-se um questionário estruturado e o mesmo entrevistador se responsabiliza por realizar a entrevista também com a mãe de um $\mathrm{RN}$ controle de acordo com os critérios já relatados. As entrevistas duravam em média 50 minutos.

O questionário continha 88 questões e foi adaptado do modelo utilizado pelo ECLAMC. Era um instrumento estruturado, com perguntas fechadas sobre três pilares: os genitores, o neonato e a gestação. Questionou-se sobre aspectos socioeconômicos dos pais, antecedentes familiares de defeito congênito e consanguinidade parental. Quanto ao $\mathrm{RN}$, foram registradas características como sexo, peso, idade gestacional, tipo de parto, apresentação fetal e definição de $\mathrm{MF}$, quando presentes. Em relação à gestação, foram investigadas, além da realização de pré-natal, diversas possíveis exposições que pudessem servir de confundidores na análise de risco do misoprostol. As principais foram: vacinas, doenças, medicamentos, álcool, tabaco, drogas ilícitas e fatores físicos. Foram ainda acrescidas questões específicas sobre planejamento e desejo da gravidez, tentativa de aborto e uso do misoprostol durante a gestação. Os questionários foram revisados imediatamente após a entrevista e comparados a outras informações oriundas de fichas de internação, prontuário médico e exames, e possíveis dúvidas eram elucidadas junto ao corpo clínico que acompanhava o caso. Os dados foram digitados em banco estruturado no Excel e duplamente checados para evitar erros de digitação.

A análise univariada se deu com a utilização das medidas estatísticas de frequência absoluta e relativa, média, mediana e desvio padrão. Para análise bivariada, com intuito de verificar a associação entre presença (caso) e ausência (controle) de MF e as principais variáveis, utilizou-se o teste não-paramétrico do $\chi^{2}$, teste de FisherFreeman-Halton e teste Z. Para todos os testes, fixou-se o nível de significância máximo de $5 \%$. Foi calculada a Odds Ratio como aproximação do risco relativo entre RN malformados e associação ao uso de misoprostol na gestação. O programa utilizado para análise foi o Statistical Package for the Social Sciences (SPSS), versão 13.0.

As parturientes foram convidadas a participarem do estudo após explicações sobre os objetivos da pesquisa e apresentação do termo de consentimento livre e esclarecido. A pesquisa teve aprovação de Comitê de Ética em Pesquisa do Hospital Geral Dr. César Cals, cadastrado no Sistema Nacional de Ética em Pesquisa.

\section{Resultados}

O total de partos nas maternidades no período estudado foi de 6.978, dos quais $126(1,8 \%)$ cumpriam os critérios para inclusão de RN como casos na amostra, tendo sido coletados também dados de 126 controles. Toda a amostra se apresentou homogênea quanto às características socioeconômicas. Em ambos os grupos, a maioria dos genitores era procedente do interior do Estado e tinha escolaridade fundamental incompleta. As mulheres estavam desempregadas ou eram do lar e os homens tinham ocupação sem qualificação formal. Setenta e oito por cento dos pais tinham união consensual, a maioria com tempo de convivência de um a cinco anos.

A Tabela 1 reúne as principais características dos $\mathrm{RN}$. As diferenças significativas encontradas entre os grupos dizem respeito aos já esperados desfechos desfavoráveis para $\mathrm{RN}$ com $\mathrm{MF}$, já que são mais suscetíveis à restrição de crescimento e comorbidades associadas frente a $\mathrm{RN}$ saudáveis.

Tabela 1 - Comparação entre os grupos caso e controle quanto às principais características dos recém-nascidos

\begin{tabular}{|c|c|c|c|c|c|c|c|}
\hline \multirow{2}{*}{ Características dos RN } & \multicolumn{2}{|c|}{ Caso } & \multicolumn{2}{|c|}{ Controle } & \multicolumn{2}{|c|}{ Total } & \multirow{2}{*}{$\begin{array}{l}\text { Valor } \\
\text { de } p\end{array}$} \\
\hline & n & $\%$ & n & $\%$ & n & $\%$ & \\
\hline Peso (gramas) & & & & & & & $0,0001^{\star}$ \\
\hline$<2.500$ & 47 & 37,3 & 21 & 16,7 & 68 & 27,0 & \\
\hline 2.500 a 4.000 & 76 & 60,3 & 97 & 77,0 & 173 & 68,7 & \\
\hline$>4.000$ & 3 & 2,4 & 8 & 6,3 & 11 & 4,4 & \\
\hline Adequação Idade Gestacional & & & & & & & $0,006^{*}$ \\
\hline PIG & 27 & 21,4 & 10 & 7,9 & 37 & 14,7 & \\
\hline AIG & 89 & 70,6 & 99 & 78,6 & 188 & 74,6 & \\
\hline GIG & 10 & 7,9 & 17 & 13,5 & 27 & 10,7 & \\
\hline Sexo & & & & & & & $0,9 \uparrow$ \\
\hline Masculino & 64 & 50,8 & 68 & 54,0 & 132 & 52,4 & \\
\hline Feminino & 56 & 44,4 & 58 & 46,0 & 114 & 45,2 & \\
\hline Intersexo & 6 & 4,8 & 0 & 0,0 & 6 & 2,4 & \\
\hline Status ao nascimento & & & & & & & $0,02^{*}$ \\
\hline Vivo & 117 & 92,9 & 125 & 99,2 & 242 & 96,0 & \\
\hline Morto & 9 & 7,1 & 1 & 0,8 & 10 & 4,0 & \\
\hline Status na alta hospitalar & & & & & & & $0,0001^{*}$ \\
\hline Vivo & 8 & 6,3 & 22 & 17,5 & 30 & 11,9 & \\
\hline Morto & 11 & 9,4 & 0 & 0,0 & 11 & 4,5 & \\
\hline Sem alta & 98 & 77,8 & 103 & 81,7 & 201 & 79,8 & \\
\hline
\end{tabular}

Valor de $\mathrm{p}$ no teste do $\chi^{2}$.

* $p$ inferior a 0,05 ; †Teste do $\chi^{2}$ excluindo a categoria intersexo.

PIG: pequenos para idade gestacional; AIG: adequados para idade gestacional; GIG: grandes para idade gestacional. 
$\mathrm{Na}$ Tabela 2, comparamos a exposição aos principais fatores de risco biológicos para malformação congênita entre os grupos. Quanto à exposição a fatores físicos, cerca de $24 \%$ de toda a amostra relatou que sofreu algum tipo de traumatismo, radiografia, procedimento invasivo e choque elétrico, embora sem consequências para a gestação. Não houve diferença significativa quando comparadas frequências de exposição física entre os grupos caso e controle $(\mathrm{p}=0,7)$.

Os medicamentos foram os principais fatores de exposição química mencionados. Do total da amostra, 210 mães $(83,3 \%)$ reportaram ter utilizado no mínimo um tipo de medicamento pelo menos uma vez durante a gestação, totalizando 383 exposições, com uma média de 1,8 medicamentos por gestante. Quando foram comparadas as frequências de uso por meio do teste $Z$, não houve diferenças de exposição entre os grupos. $\mathrm{O}$ único medicamento citado com risco $\mathrm{X}$ pela classificação da Food and Drug Administration foi o misoprostol, ou seja, medicamento contraindicado

Tabela 2 - Fatores biológicos de risco para malformações fetais nos casos e controles

\begin{tabular}{|c|c|c|c|c|c|c|c|}
\hline \multirow{2}{*}{ Fatores } & \multicolumn{2}{|c|}{ Caso } & \multicolumn{2}{|c|}{ Controle } & \multicolumn{2}{|c|}{ Total } & \multirow{2}{*}{$\begin{array}{l}\text { Valor } \\
\text { de } p\end{array}$} \\
\hline & n & $\%$ & n & $\%$ & n & $\%$ & \\
\hline Idade materna (anos) & & & & & & & 0,6 \\
\hline Até 19 & 14 & 11,1 & 21 & 16,6 & 35 & 13,9 & \\
\hline 20 a 35 & 99 & 78,6 & 93 & 73,9 & 192 & 76,2 & \\
\hline Acima de 35 & 13 & 10,3 & 12 & 9,5 & 25 & 9,9 & \\
\hline Idade paterna (anos) & & & & & & & 0,3 \\
\hline Até 39 & 106 & 85,5 & 108 & 90,0 & 214 & 87,7 & \\
\hline$\geq \mathbf{4 0}$ & 18 & 14,5 & 12 & 10,0 & 30 & 12,3 & \\
\hline Consanguinidade & & & & & & & 0,6 \\
\hline Sim & 12 & 9,5 & 9 & 7,3 & 21 & 8,4 & \\
\hline Não & 114 & 90,5 & 114 & 92,7 & 228 & 91,6 & \\
\hline História familiar de malformação & & & & & & & 0,6 \\
\hline Sim & 23 & 18,3 & 19 & 15,2 & 42 & 16,7 & \\
\hline Não & 103 & 81,7 & 106 & 84,8 & 209 & 83,3 & \\
\hline Apresentação ao nascer & & & & & & & $0,03^{*}$ \\
\hline Cefálica & 85 & 76,6 & 99 & 87,6 & 184 & 82,1 & \\
\hline Pélvica & 22 & 19,8 & 9 & 8,0 & 31 & 13,8 & \\
\hline Outra & 4 & 3,6 & 5 & 4,4 & 9 & 4,0 & \\
\hline
\end{tabular}

Valor de $p$ no teste do $\chi^{2}$

* $p$ inferior a 0,05 . na gravidez porque o risco fetal se sobrepõe claramente a qualquer possível benefício para a gestante ${ }^{14}$.

A exposição a drogas durante a gestação concentrou-se principalmente em uso de tabaco e de álcool (15,9 e 8,3\% da amostra total, respectivamente), sem diferenças significativas entre os grupos quando aplicado o teste do $\chi^{2}(\mathrm{p}=0,117)$. Duas mulheres também mencionaram consumo de maconha e crack, porém, segundo relataram, não fizeram uso de misoprostol.

O planejamento da gravidez foi relatado por $35,7 \%$ da amostra, e quase o dobro teve um sentimento positivo ao saber da gestação (68\%), não havendo diferença significativa entre os grupos. A frequência de mães que não planejaram, não desejaram a gestação e também negaram tentativa de aborto foi de $15,6 \%$, número equivalente entre os grupos.

Sobre a amostra total, 13,1\% afirmaram ter utilizado pelo menos um tipo de substância para "descer a menstruação", distribuídos em 19 casos e 14 controles, não havendo diferença entre os grupos $(\mathrm{p}=0,4)$. O uso de método abortivo durante a gestação foi declarado por 17 pacientes de uma amostra de 250 mulheres (6,8\%), visto que um caso e um controle recusaram-se a responder. Destas, dez foram no grupo caso e sete no Grupo Controle ( $\mathrm{p}=0,6)$.

O uso de misoprostol $\left(\right.$ Cytotec $\left.^{\circledR}\right)$ durante a gestação com finalidade abortiva foi relatado por 3,5\% das entrevistadas, sendo 7/119 do Grupo Caso e 2/124 do Grupo Controle; $\mathrm{OR}=3,65$ (IC95\%=0,74-17,91).

As malformações detectadas na amostra de $126 \mathrm{RN}$ classificados como casos foram ordenadas por sistema ou órgão acometido e por gravidade. No total, foram diagnosticadas 136 MF, totalizando uma média de 1,07 malformações/RN.

Mais da metade dos malformados teve diagnóstico pré-natal $(54,8 \%, n=69)$ e $34,9 \%(n=44)$ dos casos foram detectados no primeiro dia de nascimento. Apenas 10,3\% $(\mathrm{n}=13)$ tiveram diagnóstico tardio: a partir do segundo dia de nascimento, porém dentro do mesmo intervalo de tempo da internação consequente ao nascimento.

A Tabela 3 apresenta o perfil de malformações avaliadas no período do estudo dentre os $\mathrm{RN}$ que foram expostos ao misoprostol durante a gestação, bem como algumas variáveis que poderiam estar envolvidas na relação de causa e efeito.

Tabela 3 - Perfil de malformações encontradas em casos de exposição ao misoprostol durante a gestação

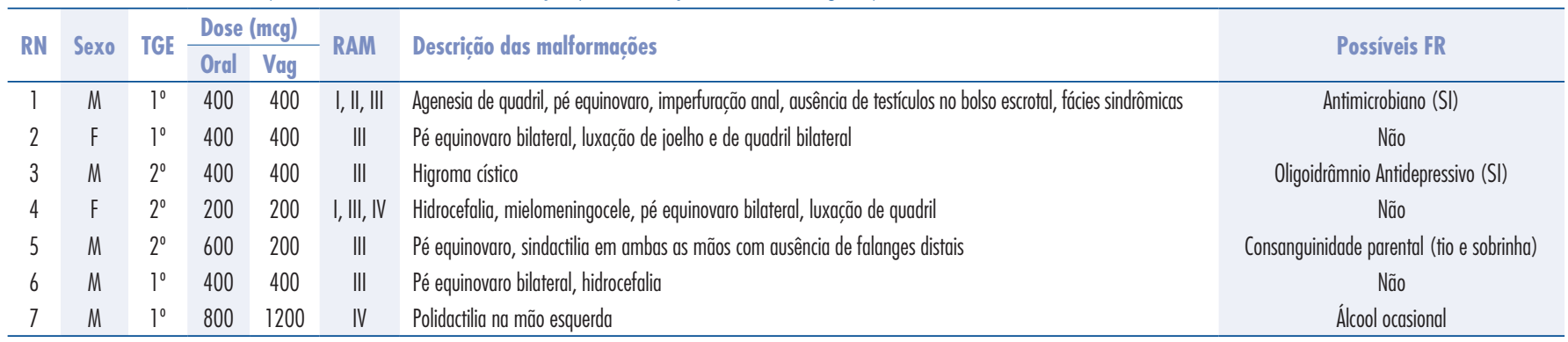

M: masculino; F: feminino; I: náuseas, II: diarreia; III: sangramento transvaginal; IV: cólica

TGE: trimestre gestacional de exposição; mcg: microgramas; Vag: vaginal; RAM: reação atribuível ao misoprostol; FR: fatores de risco; SI: sem identificação. 


\section{Discussão}

Alguns cuidados foram providenciados para tentar controlar os vieses característicos dos estudos de casocontrole. Primeiro, foram selecionados os serviços responsáveis pela maioria dos partos realizados na cidade de Fortaleza para diminuir o viés de amostragem. Depois, a amostra foi pareada segundo gênero, local e tempo de nascimento de forma a tornar os grupos mais semelhantes, sendo o diagnóstico de malformação a única característica que os diferenciou. Ainda, para minimizar possíveis erros referentes à investigação retrospectiva, no caso de viés de memória materna, as entrevistas foram conduzidas com perguntas estruturadas com o objetivo de captar o uso de misoprostol de três formas: "tomou algo para descer a menstruação?", "tentou aborto?" e "usou misoprostol?”. Da mesma forma, muitas informações oriundas das entrevistas foram confirmadas a partir dos prontuários e cartões de pré-natal das pacientes.

Entretanto, este estudo apresenta algumas limitações. A primeira diz respeito à própria temática investigada, podendo gerar omissão por parte das puérperas entrevistadas tanto pela ilegalidade do aborto quanto pelos sentimentos de culpa quanto ao diagnóstico de MF do filho. Quase 35\% dos casos tiveram diagnóstico de MF após o nascimento, o que coloca estas mães diante de uma informação recente e dramática e dificulta ainda mais o acesso a essa população. Por fim, parte dos malformados nascidos nos hospitais do estudo pode não ter sido notificada pelas equipes consultadas seja pelo desgaste ou desinteresse dos informantes ou pela falta de diagnóstico e subnotificação dos casos.

Dados do Ministério da Saúde registram 43.800 nascidos vivos na cidade de Fortaleza em 2005. Destes, somente 216 tiveram registro oficial de algum tipo de anomalia congênita ${ }^{12}$. O presente estudo abrangeu 15,9\% do total de partos realizados no serviço público na cidade de Fortaleza em 2005. No entanto, a amostra de RN malformados, excluindo-se os natimortos, compreendia $55 \%$ do total registrado oficialmente. Esses dados são conflitantes e revelam a subnotificação dos defeitos congênitos que, no Brasil, ocorrem em até $60,7 \%$ dos nascimentos, mesmo após a implementação de um campo específico para registro de $\mathrm{MF}$ na declaração de nascimento ${ }^{15}$. O acesso a tal informação poderia ampliar o conhecimento acerca da realidade brasileira sobre doenças congênitas, permitindo associá-las a possíveis riscos teratogênicos durante a gestação, como no caso do misoprostol. Este é o primeiro passo para o desenvolvimento de políticas efetivas focadas em prevenção.

No que tange às características dos $\mathrm{RN}$ investigados, as diferenças de peso, adequação de peso e idade gestacional, status ao nascimento e status na alta hospitalar encontradas entre os grupos caso e controle não interferiram na análise de causalidade de misoprostol sobre a MF, porque são eventos sabidamente decorrentes dos defeitos congênitos.

O presente estudo se baseou numa investigação multifatorial de causalidade, relacionando variáveis confundidoras, tais como fatores de risco biológicos, físicos e químicos para doença congênita. Em relação aos fatores de risco biológicos para $\mathrm{MF}$, somente a apresentação pélvica ao nascer foi superior no Grupo Caso quando comparado ao Grupo Controle. A frequência de MF é maior entre as apresentações pélvicas (varia de 6 a 18\%) e contribui significativamente para maior taxa de morbimortalidade, ocorrendo parto vaginal ou abdominal ${ }^{16}$.

No Brasil há alguns estudos que avaliam a amplitude da exposição de gestações a medicamentos ${ }^{17,18}$, o principal fator de exposição química da amostra desta pesquisa. $\mathrm{O}$ presente estudo detectou que $83,3 \%$ das mães entrevistadas referiram usar pelo menos um medicamento durante a gestação, frequência próxima à de $83,8 \%$ verificada por Mengue et al. ${ }^{19}$. Foi detectado um total de 383 exposições, perfazendo uma média de 1,8 medicamentos por gestante, inferior aos 2,3 medicamentos por gestante relatados ${ }^{17}$.

É possível que por se tratar de medicamento e, portanto, com base no ponto de vista da saúde e da indústria farmacêutica, há uma maior aceitação entre as gestantes para a utilização do misoprostol, e este pode ser um fator facilitador de seu uso nessa população para provocar aborto. Além disso, estudos indicam que as mulheres buscam os métodos abortivos mais seguros e efetivos associados a menor efeito adverso e dor, à conveniência, que não seja invasivo e que seja econômico ${ }^{20}$. Estes aspectos podem classificar o misoprostol como método de escolha para o aborto clandestino no Brasil.

Perguntas introdutórias neste trabalho objetivaram captar de forma espontânea e independente a utilização do misoprostol, como o planejamento ou desejo da gestação, uso de algo para "descer a menstruação" ou tentativa de aborto. As gestações indesejadas são mais suscetíveis à prática do aborto, embora isso não implique necessariamente a sua realização. Mesmo assim, é importante destacar que, embora tenham negado tentativa de aborto, mais de $15 \%$ das mulheres entrevistadas não planejaram nem se sentiram felizes com a notícia da gestação, e 13,1\% mencionaram ter utilizado algum método para induzir a menstruação, corroborando os $14,6 \%$ encontrados por Dal Pizzol et al. ${ }^{21}$.

O uso de misoprostol foi declarado por $53 \%$ das mulheres que mencionaram tentativa de aborto, valor próximo à taxa de quase $50 \%$ encontrada em estudo realizado no sul do Brasil $^{22}$ e de $57 \%$ no Rio de Janeiro ${ }^{23}$. No entanto, foi bem inferior ao primeiro trabalho brasileiro de 1993, que apresentou o uso de misoprostol em $75 \%$ 
das tentativas de aborto ${ }^{24}$. Isso demonstra que apesar das recorrentes restrições comerciais impostas ao medicamento no país, por mais de uma década, depois de identificado a sua utilização como abortivo, os relatos de seu uso têm diminuído, porém o misoprostol ainda é vendido de forma clandestina e utilizado com essa finalidade.

A frequência de exposição do feto ao misoprostol na literatura varia de 2,2 a 10\%, mas, na prática, devido às limitações dos estudos, pode-se vivenciar uma frequência maior ${ }^{21-23}$. Neste estudo, a despeito das possíveis limitações, 3,6\% das gestações declararam terem sido expostas ao misoprostol, com maior notificação entre as mães de RN com MF.

Em relação ao espectro de malformações relacionadas ao uso do misoprostol no presente estudo, todas as condições, com exceção do RN com higroma cístico, obedecem a um padrão de defeitos congênitos citados em artigos publicados sobre uso de misoprostol e relação com MF congênitas ${ }^{7,8}$. Além disso, defeitos congênitos como pé equinovaro, defeito de redução de membros, agenesia de falanges e sindactilia são MF que podem ser explicadas por disrupção vascular, mecanismo associado a fortes contrações uterinas e que podem ser ocasionadas pelo misoprostol ${ }^{25}$. Por fim, as MF encontradas apresentam uma relação temporal de exposição ao misoprostol e surgimento da MF e de ausência de outros fatores de risco significativos (à exceção de um caso, pela consanguinidade dos pais).

Os achados deste estudo demonstraram que, mesmo diante das restrições de uso, não existe um controle efetivo do misoprostol, utilizado ainda hoje como método abortivo no Brasil. Apesar de inúmeros estudos indicarem o uso bem sucedido do misoprostol como método abortivo, é realizado sob cuidados médicos, com doses e vias de administrações adequadas e em associação a outros medicamentos, o que contribui para sua efetividade ${ }^{26-28}$.

Ao utilizarem o misoprostol de forma clandestina, as mães correm o risco de não abortarem e gestarem filhos com doenças congênitas, transferindo os riscos que anteriormente eram delas, por se submeterem às diversas práticas abortivas, para os fetos expostos ao misoprostol, que podem nascer com danos permanentes e limitantes ${ }^{29}$. É imprescindível que mulheres em idade fértil tenham acesso à informação sobre os possíveis riscos de doenças congênitas para o feto exposto ao misoprostol em tentativas mal sucedidas de aborto ${ }^{30}$.

Apesar de limitações no poder estatístico, os resultados deste estudo sugerem uma maior exposição ao misoprostol durante a gestação em RN malformados comparados a RN saudáveis, estando os primeiros mais suscetíveis a MF congênitas limitantes. Estes dados são também apropriados para posterior incorporação em meta-análises sobre o tema.

\section{Agradecimentos}

Apoio da Fundação Cearense de Amparo à Pesquisa (FUNCAP) - Bolsa para o Pesquisador principal.

Aos acadêmicos de Medicina pela coleta dos dados: André Alcântara, Carolina Arcanjo, Kathiane Lustosa, Leonardo Costa, Marcelle Parente, Nádia Viana, Renato Albano e Sara Arcanjo; à Milena Portela pela organização dos dados e treinamento da geneticista Dra. Erlane Marques Ribeiro.

\section{Participação dos autores}

Emérita Opaleye elaborou o projeto, coordenou a coleta de dados, discutiu os resultados e redigiu o artigo. Elaine Santos e Ana Júlia Ribeiro participaram da coleta e da organização dos dados. Helena Coelho e Lavínia Schüler-Faccini participaram da elaboração do projeto, discussão dos resultados e revisão do manuscrito. Paulo Almeida realizou as análises estatísticas e discutiu os resultados. Fabrício Costa desenhou o estudo, participou da elaboração do projeto, discutiu os resultados, idealizou e efetuou a revisão final do manuscrito.

\section{Referências}

1. Monteiro MFG, Adesse L. Estimativas de aborto induzido no Brasil e Grandes Regiões (1992-2005) [Internet]. 2006 [citado 2010 Jan 19]. Disponível em: http://www.ipas.org.br/arquivos/ ml2006.pdf

2. Costa SH. Commercial availability of misoprostol and induced abortion in Brazil. Int J Gynaecol Obstet. 1998;63 Suppl $1:$ S131-9.

3. Coelho HL, Teixeira AC, Cruz MF, Gonzaga SL, Arrais PS, Luchini $\mathrm{L}$, et al. Misoprostol: the experience of women in Fortaleza, Brazil. Contraception. 1994;49(2):101-10.
4. Guest J, Chien PF, Thomson MA, Kosseim ML. Randomised controlled trial comparing the efficacy of same-day administration of mifepristone and misoprostol for termination of pregnancy with the standard 36 to 48 hour protocol. BJOG. 2007; 1 14(2): 207-15.

5. Shannon C, Wiebe E, Jacot F, Guilbert E, Dunn S, Sheldon WR, et al. Regimens of misoprostol with mifepristone for early medical abortion: a randomised trial. BJOG. 2006;113(6):621-8.

6. Song J. Use of misoprostol in obstetrics and gynecology. Obstet Gynecol Surv. 2000;55(8):503-10. 
7. Da Silva Dal Pizzol T, Knop FP, Mengue SS. Prenatal exposure to misoprostol and congenital anomalies: systematic review and meta-analysis. Reprod Toxicol. 2006;22(4):666-71.

8. Gonzalez CH, Marques-Dias M, Kim CA, Sugayama SM, Da Paz JA, Huson SM, et al. Congenital abnormalities in Brazilian children associated with misoprostol misuse in first trimester of pregnancy. Lancet. 1998;351(91 16):1624-7.

9. Pastuszak AL, Schüler L, Speck-Martins CE, Coelho KE, Cordello SM, Vargas $F$, et al. Use of misoprostol during pregnancy and Möbius' syndrome in infants. N Engl J Med. 1998;338(26):1881-5.

10. Schüler L, Pastuszak AL, Sanseverino MT, Oriolli IM, Brunoni $D$, Ashton-Prolla $P$, et al. Pregnancy outcome after exposure to misoprostol in Brazil: a prospective, controlled study. Reprod Toxicol. 1999; 13(2): 147-51.

11. Da Silva Dal Pizzol T, Tierling VL, Schüler-Faccini L, Sanseverino MT, Mengue SS. Reproductive results associated with misoprostol and other substances utilized for interruption of pregnancy. Eur J Clin Pharmacol. 2005;61(1):71-2.

12. Brasil. Ministério da Saúde. Sistema de Informações de Nascidos Vivos (SINASC) [Internet]. [citado 2010 Jan 19]. Disponível em: http://portal.saude.gov.br/portal/saude/visualizar_texto. cfm? idtxt=2 1379

13. Estudo Colaborativo Latino Americano de Malformações Congênitas (ECLAMC). Manual operacional ECLAMC. 2a ed. Rio de Janeiro: ECLAMC; 2002.

14. Briggs GG, Freeman RK, Yaffe SJ. Drugs in pregnancy and lactation. 6a ed. Philadelphia: Lippincott Williams \& Wilkins; 2002.

15. Luquetti DV, Koifman RJ. Quality of reporting on birth defects in birth certificates: case study from a Brazilian reference hospital. Cad Saúde Pública. 2009;25(8):1721-31.

16. Wester R. Apresentação pélvica. In: Frederickson HL, WilkinsHaug L. Segredos em ginecologia e obstetrícia. 2a ed. Porto Alegre: Artmed; 2000. p. 359-64.

17. Osorio-de-Castro CGS, Pepe VLE, Luiza VL, Cosendey MAE, Freitas AM, Miranda FF, et al. Uso indicado e uso referido de medicamentos durante a gravidez. Cad Saúde Pública. 2004;20 Supl 1:S73-S82.
18. Fonseca MRCC, Fonseca E, Bergsten-Mendes G. Prevalência do uso de medicamentos na gravidez: uma abordagem farmacoepidemiológica. Rev Saúde Pública. 2002;36(2):205-12.

19. Mengue SS, Schenkel EP, Duncan BB, Schmidt MI. Uso de medicamentos por gestantes em seis cidades brasileiras. Rev Saúde Pública. 2001;35(5):415-20.

20. Bartz D, Goldberg A. Medication abortion. Clin Obstet Gynecol. 2009;52(2): 140-50.

21. Dal Pizzol TS, Sanseverino MTV, Mengue SS. Exposure to misoprostol and hormones during pregnancy and risk of congenital anomalies. Cad Saúde Pública. 2008;24(6): 1447-53.

22. Fonseca $W$, Misago C, Freitas $P$, Santos E, Fernandes L, Correia L. Características sócio-demográficas, reprodutivas e médicas de mulheres admitidas por aborto em hospital da região sul do Brasil. Cad Saúde Pública. 1998;14(2):279-86.

23. Costa SH, Vessey MP. Misoprostol and illegal abortion in Rio de Janeiro, Brazil. Lancet. 1993;341(8855):1258-61.

24. Coelho HL, Teixeira AC, Santos AP, Forte EB, Morais SM, La Vecchia $C$, et al. Misoprostol and illegal abortion in Fortaleza, Brazil. Lancet. 1993;341(8855):1261-3.

25. Shepard TA. Möbius syndrome after misoprostol: a possible teratogenic mechanism. Lancet. 1995;346(8977):780.

26. Salakos N, lavazzo C, Bakalianou K, Gregoriou O, Paltoglou G, Kalmantis K, et al. Misoprostol use as a method of medical abortion. Clin Exp Obstet Gynecol. 2008;35(2):130-2.

27. Gemzell-Danielsson K, Lalitkumar S. Second trimester medical abortion with mifepristone-misoprostol and misoprostol alone: a review of methods and management. Reprod Health Matters. 2008;16(31 Suppl): 162-72.

28. Lohr PA, Hayes JL, Gemzell-Danielsson K. Surgical versus medical methods for second trimester induced abortion. Cochrane Database Syst Rev. 2008;(1):CD006714.

29. Mengue SS, Dal Pizzol TS. Misoprostol, aborto e malformações congênitas. Rev Bras Ginecol Obstet. 2008;30(6):271-3.

30. Silva MBS, Ferreira AA, Habr SF, Bernardi MM. Misoprostol and teratogenesis in neonates. Braz J Pharm Sci. 2009;45(3):417-2. 